\title{
Ginsenoside 20(S)-Rg3 Inhibits the Warburg Effect Via Modulating DNMT3A/ MiR-532-3p/HK2 Pathway in Ovarian Cancer Cells
}

\author{
Yuanyuan Zhou Xia Zheng $^{a, b} \quad$ Jiaojiao Lu ${ }^{a, b}$ Wei Chen ${ }^{c} \quad$ Xu Lia,b Le Zhao ${ }^{a, b}$ \\ aCenter for Translational Medicine, the First Affiliated Hospital of Xi'an Jiaotong University, Xi'an, ${ }^{\mathrm{b} K e y}$ \\ Laboratory for Tumor Precision Medicine of Shaanxi Province, the First Affiliated Hospital of Xi'an \\ Jiaotong University, Xi'an, 'Center for Laboratory Medicine, the First Affiliated Hospital of Xi'an Jiaotong \\ University, Xi'an, P.R. China
}

\section{Key Words}

Ginsenoside • Ovarian cancer • Warburg effect • MicroRNA

\begin{abstract}
Background/Aims: The Warburg effect is one of the main energy metabolism features supporting cancer cell growth. 20(S)-Rg3 exerts anti-tumor effect on ovarian cancer partly by inhibiting the Warburg effect. microRNAs are important regulators of the Warburg effect. However, the microRNA regulatory network mediating the anti-Warburg effect of 20(S)-Rg3 was largely unknown. Methods: microRNA deep sequencing was performed to identify the 20(S)-Rg3-influenced microRNAs in SKOV3 ovarian cancer cells. miR-532-3p was overexpressed by mimic532-3p transfection in SKOV3 and A2780 cells or inhibited by inhibitor532-3p transfection in 20(S)-Rg3-treated cells to examine the changes in HK2 and PKM2 expression, glucose consumption, lactate production and cell growth. Dual-luciferase reporter assay was conducted to verify the direct binding of miR-532-3p to HK2. The methylation status in the promoter region of pre-miR-532-3p gene was examined by methylation-specific PCR. Expression changes of key molecules controlling DNA methylation including DNMT1, DNMT3A, DNMT3B, and TET1-3 were examined in 20(S)-Rg3-treated cells. DNMT3A was overexpressed in 20(S)-Rg3-treated cells to examine its influence on miR-532-3p level, HK2 and PKM2 expression, glucose consumption and lactate production. Results: Deep sequencing results showed that 11 microRNAs were increased and 9 microRNAs were decreased by 20(S)Rg3 in SKOV3 cells, which were verified by qPCR. More than 2-fold increase of miR-532-3p was found in 20(S)-Rg3-treated SKOV3 cells. Forced expression of miR-532-3p reduced HK2 and PKM2 expression, glucose consumption and lactate production in SKOV3 and A2780 ovarian cancer cells. Inhibition of miR-532-3p antagonized the suppressive effect of 20(S)-Rg3 on HK2 and PKM2 expression, glucose consumption and lactate production in ovarian cancer cells.
\end{abstract}

$Y$. Zhou and $X$. Zheng contributed equally to this work.

Le Zhao, Ph.D. and Xu Li, Ph.D.
Center for Translational Medicine, Key Laboratory for Tumor Precision Medicine of Shaanxi Province, the First Affiliated Hospital of Xi'an Jiaotong University, Xi'an, 710061, (P.R. China); E-Mail zhaole2@mail.xjtu.edu.cn, lixu56@mail.xjtu.edu.cn 
Zhou et al.: 20(S)-Rg3 Inhibits the Warburg Effect in Ovarian Cancer

Dual-luciferase reporter assay showed that miR-532-3p directly suppressed HK2 rather than PKM2. miR-532-3p level was controlled by the methylation in the promoter region of its host gene. 20(S)-Rg3 inhibited DNMT3A expression while exerted insignificant effect on DNMT1, DNMT3B and TET1-3. 20(S)-Rg3 reversed DNMT3A-mediated methylation in the promoter of the host gene of miR-532-3p, and thus elevated miR-532-3p level followed by suppression of HK2 and PKM2 expression, glucose consumption and lactate production. Conclusions: 20(S)-Rg3 modulated microRNAs to exert the anti-tumor effect in ovarian cancer. 20(S)-Rg3 lessened the DNMT3A-mediated methylation and promoted the suppression of miR-532-3p on HK2 to antagonize the Warburg effect of ovarian cancer cells.

\section{Introduction}

Ovarian cancer is the most lethal gynecological malignancy. Most patients are diagnosed with advanced stage disease at presentation [1]. Despite advances in treatment, mortality rate of patients suffered advanced ovarian cancer remains high, leaving an urgent need for new treatment strategies.

Aberrant energy metabolism has been listed as one of the hallmarks of cancers, among which the reprogramming of glucose metabolism is the pivotal feature, promising a class of therapeutic targets for cancer [2,3]. In cancer cells, glucose is preferentially metabolized by aerobic glycolysis than by mitochondrial oxidative phosphorylation, which is dubbed the Warburg effect [4]. The Warburg effect is catalyzed by several key glycolytic enzymes including hexokinase-2 (HK2), phosphofructokinase (PFK)-1, pyruvate kinase M2 (PKM2), pyruvate dehydrogenase kinase-1 (PDK-1), and lactate dehydrogenase (LDH) [5]. Of note, HK2 catalyzes the essentially irreversible first step of the glycolytic pathway in which glucose is phosphorylated to glucose-6-phosphate with concomitant dephosphorylation of ATP. The Warburg effect not only allows cancer cells to meet their high energy demands and supply the anabolic precursors for macromolecules, but also minimizes reactive oxygen species production in mitochondria, thereby providing a growth advantage for cancer cells [6].

Ginsenosides are the pharmacologically active components of Panax ginseng, which has long been utilized as a traditional Chinese medicine for officinal or recuperative purposes [7]. We have reported that $20(\mathrm{~S})-\mathrm{Rg} 3$, one of the bioactive extracts from ginsenosides, reversed the vicious phenotype of ovarian cancer cells partly via inhibiting the Warburg effect $[8,9]$. The preliminary study has found that 20(S)-Rg3 inhibits the Warburg effect via STAT3/HK2 pathways [10].

Recently, microRNAs (miRs) have emerged as a new class of regulators of the Warburg effect $[11,12]$. miRs are a class of small (19-25 nucleotides) non-coding RNAs, binding messenger RNAs at their 3'-untranslated regions (3'-UTRs) to suppress their targets expression either by inhibiting protein translation or causing mRNA cleavage [13]. Increasing anti- or pro-Warburg effect miRs have been identified [14-17]. However, the miR-regulatory network mediating the anti-Warburg effect of 20(S)-Rg3 has yet to be revealed.

In the present study, we discovered that a few miRs were influenced by $20(\mathrm{~S})$-Rg3 by means of deep sequencing technique and qPCR, among which miR-532-3p inhibited aerobic glycolysis in ovarian cancer cells by directly targeting HK2. We also found that miR-532$3 p$ was negatively regulated by DNMT3A-meidated DNA methylation. The specific role of $\mathrm{miR}-532-3 \mathrm{p}$ in the Warburg effect of ovarian cancer acquired in our study will enlarge the theoretical understanding of the glucose metabolism reprogramming in human ovarian cancer and provide potential therapeutic targets to treat the disease.

\section{Materials and Methods}

Cell lines and cell culture

The human ovarian cancer cell line SKOV3 was obtained from the Shanghai Cell Bank of Chinese Academy of Sciences (Shanghai, China), and A2780 was from the Shandong Academy of Medical Sciences 


\section{Cellular Physiology Cell Physiol Biochem 2018;45:2548-2559 and Biochemistry Published onlIne: March 20, 2018 \begin{tabular}{l|l} 
DOI: 10.1159/000488273 & $\begin{array}{l}\text { (c) } 2018 \text { The Author(s). Published by S. Karger AG, Basel } \\
\text { www.karger.com/cpb }\end{array}$
\end{tabular}}

Zhou et al.: 20(S)-Rg3 Inhibits the Warburg Effect in Ovarian Cancer

(Jinan, China). Cells were cultured in RPMI 1640 medium (Gibco-BRL; Grand Island, NY Gaithersburg, USA) supplemented with 10\% fetal bovine serum (Gibco-BRL; Grand Island, NY Gaithersburg, USA) and incubated at $37^{\circ} \mathrm{C}$ in a humidified $5 \% \mathrm{CO}_{2}$.

\section{Reagents and antibodies}

Ginsenoside 20(S)-Rg3 was obtained from Tasly Pharmaceutical Company (Tianjin, China). 20(S)$\mathrm{Rg} 3$ was dissolved at a concentration of $4 \mathrm{mg} / \mathrm{mL}$ in DMSO as a stock solution and stored at $-20^{\circ} \mathrm{C}$. The concentrations of working solution of $20(\mathrm{~S})-\mathrm{Rg} 3$ was $80 \mu \mathrm{g} / \mathrm{mL}$ for SKOV3 cells and $40 \mu \mathrm{g} / \mathrm{mL}$ for A2780 cells, and the maximum final concentration of DMSO was less than $0.1 \%$. The following primary antibodies were used, $\beta$-actin, HK2, PKM2, PFK2, LDHA, DNMT3A and DNMT3B (Cell Signaling Technology; Beverly, MA, USA), Glut1 and PDK1 (Santa Cruz Biotechnology; Santa Cruz, CA, USA), DNMT1 (Active Motif; Carlsbad, CA, USA), TET1 and TET2 (Genetex Inc.; Irvine, CA, USA), TET3 (Abcam Inc.; Cambridge, MA, USA).

microRNA deep sequencing and bioinformatics analysis

Total RNAs were extracted from SKOV3 cells treated with 0 or $80 \mu \mathrm{g} / \mathrm{mL} 20(\mathrm{~S})$-Rg3 for 24h by TRIzol reagent (Invitrogen; Carlsbad, CA, USA). microRNA was purified using miRNeasy Mini Kit (Qiagen; Hilden, Germany), and the complementary DNA libraries for single-end sequencing were prepared using Ion Total RNA-Seq Kit v2.0 (ThermoFisher Scientific Inc.; Waltham, MA, USA). Sequencing was performed on the Ion Torrent Proton platform, and the microRNA deep sequencing data were analyzed by Novel Bioinformatics Ltd. Co. (Shanghai, China). Data filtering process was as follows: domestic java code was applied to filter the raw data under following criteria: 1 . Right adaptor was removed; 2 . Reads length lower than $17 \mathrm{bp}$ or larger than $33 \mathrm{bp}$ was removed after adaptor filtering; 3 . Reads with $20 \%$ base quality lower than 17 were removed. 4. Reads end with quality lower than 15 was trimmed. Clean data was obtained after data filtering. DNA mapping was performed utilizing BWA-ALN (version: 0.7.8-r455) (https://github.com/lh3/bwa) following the parameter (bwa aln -n 0.04 -e 3 -t 8) towards the human miRBase 21.0 database (http://www.mirbase. org/). miRNA counts of mature miRNA were calculated based on the bam file. EB-Seq algorithm was applied to filter the differentially expressed microRNAs, after the significant analysis and FDR analysis under the following criteria: i) Fold Change $>2$ or $<0.5$; ii) p-value $<0.05$; iii) FDR $<0.05$. The potential targets of the differentially expressed microRNAs were predicted using the miRanda algorithm [9]. Gene Ontology analysis was used to categorize the biological implications of the targets of the differentially expressed miRs. The Kyoto Encyclopaedia of Genes and Genomes (KEGG) pathway analysis was performed to identify the main biochemical and signaling pathways associated with the potential targets of the differential microRNAs.

\section{Real-time quantification of miR}

Total RNA was extracted from cells using TRIzol reagent (Invitrogen; Carlsbad, CA, USA) according to the manufacturer's instructions. Concentration and quality of total RNA were measured by a UV spectrophotometer (Bio-Rad Inc.; Hercules, CA, USA). $1 \mu \mathrm{g}$ RNA was reverse transcripted using a RevertAid First Strand cDNA Synthesis Kit (Thermo Fisher Scientific Inc., Waltham, MA, USA). Quantitative real-time PCR was performed using the SYBR Premix Ex Taq ${ }^{\text {TM }}$ II kit (Takara; Dalian, China) on a CFX96 real-time PCR system (Bio-Rad Inc.; Hercules, CA, USA). Primers for the differentially expressed microRNAs and U6 reverse transcription and amplification were designed and synthesized by Ribo-Bio Co., Ltd. (Guangzhou, China). U6 served as an internal reference for microRNA. Relative gene expression was calculated automatically using $2^{-\Delta \Delta C \mathrm{t}}$ method.

\section{MicroRNA mimic or inhibitor transfection}

miR-532-3p mimic, inhibitor and corresponding negative controls were purchased from Ribo-Bio Co. Ltd. (Guangzhou, China). $3 \times 10^{5}$ SKOV3 and A2780 cells were seeded into 6-well plates to reach 30-50\% confluency after $24 \mathrm{~h}$. $80 \mathrm{nM}$ miR-532-3p mimic and the negative controls were transiently transfected into cells using X-treme GENE siRNA Transfection Reagent (Roche; Indianapolis, IN, USA). $100 \mathrm{nM}$ inhibitor and the negative controls were transfected into cells using X-treme GENE siRNA Transfection Reagent (Roche; Indianapolis, IN, USA) for $24 \mathrm{~h}$, followed by $20(\mathrm{~S})$-Rg3 treatment for $24 \mathrm{~h}$ (for RNA extraction) or $48 \mathrm{~h}$ ( for total protein extraction).

Plasmid transfection

The human DNMT3A expression vector pcDNA3/Myc-DNMT3A was a gift from Arthur Riggs [18] (Addgene plasmid \# 35521). SKOV3 and A2780 cells were seeded into 6-well plates to reach the 70-90\% 


\section{Cellular Physiology Cell Physiol Biochem 2018;45:2548-2559 \\ \begin{tabular}{ll|l} 
and Biochemistry & $\begin{array}{l}\text { DOI: 10.1159/000488273 } \\
\text { Published online: March 20, } 2018\end{array}$ & $\begin{array}{l}\text { (c) } 2018 \text { The Author(s). Published by S. Karger AG, Basel } \\
\text { www.karger.com/cpb }\end{array}$ \\
\hline
\end{tabular}}

Zhou et al.: 20(S)-Rg3 Inhibits the Warburg Effect in Ovarian Cancer

confluency after $24 \mathrm{~h} .2 \mu \mathrm{g}$ of pcDNA3/Myc-DNMT3A or empty vector were transiently transfected into cells using the X-treme GENE HP DNA Transfection Reagent (Roche; Indianapolis, IN, USA) for 24h, followed by treatment with $20(\mathrm{~S})-\mathrm{Rg} 3$ for another $24 \mathrm{~h}$ (for total RNA extraction) or $48 \mathrm{~h}$ (for protein extraction).

Quantitative real-time polymerase chain reaction ( $q R T-P C R$ )

Total RNA was extracted from cells using TRIzol reagent (Invitrogen; Carlsbad, CA, USA) according to the manufacturer's instructions. Concentration and quality of total RNA were measured by a UV spectrophotometer (Bio-Rad Inc.; Hercules, CA, USA). $1 \mu \mathrm{g}$ RNA was used for reverse transcription by the Prime Script ${ }^{\mathrm{TM}}$ RT reagent Kit with gDNA Eraser (Takara; Dalian, China). Quantitative real-time PCR was performed using the SYBR Premix Ex Taq ${ }^{\mathrm{TM}}$ II kit (Takara; Dalian, China) on a CFX96 real-time PCR system (Bio-Rad Inc.; Hercules, CA, USA). The primers used were as follows: $\beta$-actin, 5'-TCCCTGGAGAAGAGCTACGA-3' (forward), 5'-AGCACTGTGTTGGCGTACAG-3' (reverse); DNMT3A, 5'-TATTGATGAGCGCACAAGAGAGC-3' (forward), 5'-GGGTGTTCCAGGGTAACATTGAG-3' (reverse). $\beta$-actin served as an internal reference for mRNA. Relative gene expression was calculated automatically using $2^{-\Delta \Delta \mathrm{Ct}}$ method.

\section{Western blotting}

Whole-cell extracts were prepared using RIPA lysis buffer supplemented with protease inhibitor cocktail (Roche; Indianapolis, IN, USA) and $1 \mathrm{mM}$ PMSF on ice. Protein quantification was performed using a BCA Kit (Pierce; Rockford, IL, USA). Protein lysate was separated on 10\% SDS-PAGE gels by electrophoresis and subsequently electrotransferred to a nitrocellulose membrane (Pall Life Science; Port Washington, NY, USA). After 5\% non-fat milk blocking at room temperature for $1 \mathrm{~h}$, the membranes were incubated at $4{ }^{\circ} \mathrm{C}$ overnight with the primary antibodies: rabbit anti-human HK2(1:1000), PKM2(1:10000), PFK2 (1:500), LDHA (1:1000), GLUT1 (1:200), PDK 1(1:500), DNMT3A (1:500), DNMT3B (1:500), DNMT1(1:1000), and mouse anti-human $\beta$-actin (1:1000). After washing with $0.5 \%$ TBST, the blots were incubated with HRPconjugated goat anti-rabbit or goat anti-mouse secondary antibodies for $1 \mathrm{~h}$ at room temperature. Using ECL reagents (Millipore; Billerica, MA, USA), the imaging and quantification of target proteins was obtained by Image-lab software for ChemiDoc XRS system (Bio-Rad Inc., Hercules, CA, USA).

\section{Cytotoxicity assay}

The cytotoxicity assay was performed using the cell counting kit-8 (CCK8) (7seapharmtech Co. Ltd.; Shanghai, China). A2780 cells (5000 cells per well) were cultured in a 96 -well plate for $24 \mathrm{~h}$ followed by treatment with different concentrations of $20(\mathrm{~S})$-Rg3 for another $24 \mathrm{~h}$ or $48 \mathrm{~h}$. $10 \mu \mathrm{l}$ of CCK8 was then added into each well and incubated for $2 \mathrm{~h}$ at $37^{\circ} \mathrm{C}$. Cell viability was assessed by absorbance at $450 \mathrm{~nm}$ measured on an EnSpire multimode plate reader (PerkinElmer; Waltham, MA, USA). The optical density (OD) values were input into the Graphpad Prism 6.0 software to generate a dose-response curve and calculate the IC50 value of 20 (S)-Rg3 for A2780 cells. Each concentration was measured in triplicate wells on the same plate in three independent experiments.

\section{Cell growth examination}

SKOV3 and A2780 cells (2500 cells per well) were seeded in 96-well plates for $24 \mathrm{~h}$. After reaching a confluency of 30-50\%, the cells were transfected with $80 \mathrm{nM}$ miR-532-3p mimic or the negative control by X-treme GENE siRNA Transfection Reagent (Roche; Indianapolis, IN, USA) for 5 days. Or the cells were transfected with $100 \mathrm{nM}$ miR-532-3p inhibitor for $24 \mathrm{~h}$ followed by treatment of 20(S)-Rg3 for another 5 day. The cell viability was assessed using CCK8 reagent every $24 \mathrm{~h}$. The optical density (OD) values at 450 nm were input into the Graphpad Prism 6.0 software to generate a growth curve.

\section{Measurement of glucose consumption and lactate production}

Cells were seeded into 6-well plates and treated accordingly. The supernatant was collected and the live cells were counted. The glucose concentration and lactate production in the supernatant were measured using a glucose assay kit and a lactate assay colorimetric kit (Nanjing Jiancheng Bioengineering Institute; Nanjing, China) according to the manufacturer's instructions. The optical density at $505 \mathrm{~nm}$ for glucose measurement and $530 \mathrm{~nm}$ for lactate measurement were read on an EnSpire multimode plate reader (PerkinElmer; Waltham, MA, USA). Glucose consumption and lactate production rates were calculated based on the standard curve and normalized to the cell numbers.

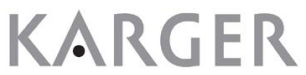




\section{Cellular Physiology Cell Physiol Biochem 2018;45:2548-2559 \\ and Biochemistry Published onlıne: March 20, $2018 \quad \begin{aligned} & \text { DOI: 10.1159/000488273 } 2018 \text { The Author(s). Published by S. Karger AG, Basel } \\ & \text { www.karger.com/cpb }\end{aligned}$}

Zhou et al.: 20(S)-Rg3 Inhibits the Warburg Effect in Ovarian Cancer

DNA bisulfite modification and methylation specific PCR (MSP)

CellsweresuspendedincoldPBSattheconcentrationof $\sim 8 \times 10^{6}$ cells $/ \mathrm{mL} .8 \times 10^{4}$ cellsweretakingtoperform bisulfite modification and purification with an EZ DNA methylation-Direct kit (Zymo Research Corporation; Irvine, California, USA) according to its instructions. The DNA concentration was assessed by absorbance at $260 \mathrm{~nm}$ on a UV spectrophotometer (Bio-Rad Inc.; Hercules, CA, USA). The promoter region of the pre-miR532-3p was set on the $2 \mathrm{~kb}$ upstream from the host gene CLCN5 sequence. The primers for methylation-specific PCR was designed using MethPrimer online tool and the sequences were as follows: methylated (M) forward: 5'-TTTAGTATTGGGATAGGATTCGTTC-3', methylated (M) reverse: 5'-TACCAAAAAAAATTTCAACTTACGC-3'; unmethylated (U) forward: 5'-GTTTAGTATTGGGATAGGATTTGTTTG-3', unmethylated (U) reverse: 5'-ACCAAAAAAAATTTCAACTTACACA-3'. MSP was performed using Epi Taq HS (Takara Biotechnology Co. Ltd.; Dalian, China) under the following condition: $94^{\circ} \mathrm{C}$ for $5 \mathrm{~min}$; followed by 40 cycles of $94{ }^{\circ} \mathrm{C}$ for $45 \mathrm{~s}$, $59^{\circ} \mathrm{C}$ for $45 \mathrm{~s}, 72^{\circ} \mathrm{C}$ for $1 \mathrm{~min}$ for M primers, or 35 cycles of $94^{\circ} \mathrm{C}$ for $45 \mathrm{~s}, 55^{\circ} \mathrm{C}$ for $45 \mathrm{~s}, 72^{\circ} \mathrm{C}$ for $1 \mathrm{~min}$ for $\mathrm{U}$ primers, followed by $72{ }^{\circ} \mathrm{C}$ for $10 \mathrm{~min}$. The PCR products were separated by $3 \%$ agarose gel electrophoresis and visualized by Image-lab software system (Bio-Rad Inc.; Hercules, CA, USA).

\section{Luciferase reporter plasmids construction}

The wild-type 3'-UTR sequence of the human HK2 gene containing two predicted miR-532-3p-binding sites were amplified by PCR from genomic DNA of SKOV3 cells using the following primers: HK2-WT 3'UTR forward: 5'- CTAATGAAGATACGGTTGC-3' and reverse: 5'- GTAGATGAGGTTAAAGAGGG-3', and another HK2-WT 3'UTR forward: 5'- GTTTCACCTTGGGTTTGT-3' and reverse: 5'- ATCCAGGTTTAATGTCTGTG-3'. The PCR products were digested with Hind III and Spe I enzymes and inserted into pMIR-REPORT luciferase vectors (Ambion; Austin, TX, USA) to construct HK2-WT-3'UTR vectors. To generate HK2 mut-3' UTR vectors, the transversion mutants in sites complementary to the miR-532-3p seed region were introduced by overlap extension method. The primers were used as follows: HK2-MUT 3'UTR P1: 5'-GGACTAGTCCGTTTCACCTTGGGTTTGT-3'; P2: 5'- TATTTCAAACTATGTTTCTGGATGAGAGTGGC-3'; P3: 5'-GCCACTCTCATCCAGAAACATAGTTTGAAATA-3'; P4: 5'-CCCAAGCTTGGGATCCAGGTTTAATGTCTGTG-3'. All the recombinant constructs were verified by DNA sequencing.

\section{Luciferase reporter assay}

Cells were seeded into a 24-well plate to reach a confluency of 80-90\%. pRL-TK vector (20 ng) and wild-type or mutation reporter vectors (180 ng) were co-transfected with $20 \mathrm{nM}$ of miR-532-3p mimic or negative control using X-treme GENE siRNA Transfection Reagent. 24h after transfection, the relative firefly luciferase activity (normalized to Renilla luciferase activity) was measured using a dual-luciferase reporter gene assay system (Promega; Madison, WI, USA), and the results were depicted as the percentage changes over the corresponding control.

\section{Statistical analysis}

Data are presented as the means \pm SE and were analyzed using SPSS 22.0 software (Chicago, IL, USA). Statistical differences were calculated by 2-tailed unpaired Student t test, or Fisher exact test for MSP results. Differences were considered significant when $\mathrm{P}<0.05\left(^{*}\right)$.

\section{Results}

Differential expression of microRNAs in the 20(S)-Rg3-treated SKOV3 cells

microRNA deep sequencing was used to examine the 20(S)-Rg3-influenced microRNAs in SKOV3 ovarian cancer cells. A total of 1, 288 microRNAs were detected in both 20(S)-Rg3treated SKOV3 cells and negative control cells. Compared to the control cells, 11 microRNAs were significantly increased and 9 microRNAs were significantly decreased in the 20(S)Rg3-treated SKOV3 cells (fold change $>1.5$ or $<0.5$, p $<0.05$ ) (Fig. 1A and 1B). The fold change (FC) and the corresponding false discovery rate (FDR) for the differentially expressed microRNAs between 20(S)-Rg3-treated and non-treated SKOV3 cells were summarized in Table 1. miR-3163, miR-664a-5p, miR-6717-5p, miR-4329, miR-603, miR-324-5p, miR-1283, miR-532-3p, miR-33a-3p, miR-519a-5p, and miR-486-3p were upregulated over 1.5 -fold in 
Fig. 1. The expression patterns of 20 differentially expressed miRs in RNA-seq. (A) Hierarchical clustering of 20 differentially expressed microRNAs in the $20(\mathrm{~S})$-Rg3-treated SKOV3 cells versus negative control cells were displayed in a heatmap (fold change of $>1.5$ or $<0.5$, FDR $<0.05$ ). Each column represented one library, and the color bar indicated the relative expression level from low (green) to high (red). EXP=20(S)-Rg3treated SKOV3 cells. $\mathrm{NC}=$ negative control SKOV3 cells. (B) The expression $\log _{2} \mathrm{FC}$ (fold

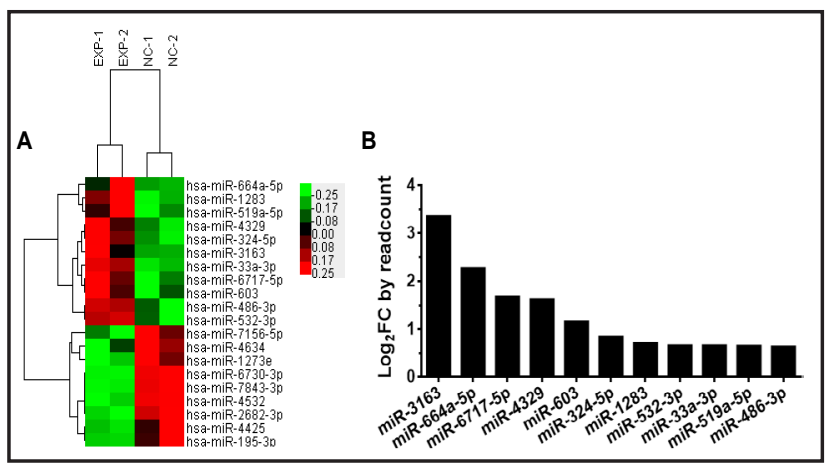
change, FC) values in the 20(S)-Rg3-treated SKOV3 cells versus negative control cells detected by RNA-seq were calculated by readcount.

the 20(S)-Rg3-treated group, while miR-4634, miR-1273e, miR-4532, miR-7156-3p, miR6730-3p, miR-2682-3p, miR-7843-3p, miR$195-3 p$, and miR-4425 were reduced in the 20(S)-Rg3-treated group to less than half the level of the control cells (Fig. 1B, Table 1).

Bioinformatics analysis of the predicted target genes of the differentially expressed microRNAs

By use of miRanda algorithm, a total of 24595 genes were predicted to be the targets of all 20 differentially expressed miRNAs. The putative target genes were involved in different biological processes such as phosphorylation and regulation of transcription shown by GO annotation (Fig. 2A). KEGG pathway analysis indicated that the predicted target genes were involved in 25 signaling pathways including

Table 1. Differentially expressed microRNAs between 20(S)-Rg3-treated and non-treated SKOV3. FC=fold change. FDR=false discovery rate

\begin{tabular}{cccc}
\hline ID & FC & FDR & Style \\
\hline hsa-miR-3163 & 10.27934 & 0.006529 & up \\
hsa-miR-664a-5p & 4.823082 & 0.030801 & up \\
hsa-miR-6717-5p & 3.198459 & 0.00156 & up \\
hsa-miR-4329 & 3.078698 & 0.002349 & up \\
hsa-miR-603 & 1.3785 & $1.29 \mathrm{E}-05$ & up \\
hsa-miR-324-5p & 1.799521 & $8.62 \mathrm{E}-06$ & up \\
hsa-miR-1283 & 1.629703 & $8.88 \mathrm{E}-16$ & up \\
hsa-miR-532-3p & 1.579486 & $1.05 \mathrm{E}-06$ & up \\
hsa-miR-33a-3p & 1.578077 & $3.96 \mathrm{E}-14$ & up \\
hsa-miR-519a-5p & 1.570293 & 0.013483 & up \\
hsa-miR-486-3p & 1.54716 & $1.9 \mathrm{E}-05$ & up \\
hsa-miR-4634 & 0.495503 & 0.020588 & down \\
hsa-miR-1273e & 0.489096 & $2.81 \mathrm{E}-06$ & down \\
hsa-miR-4532 & 0.287307 & 0 & down \\
hsa-miR-7156-5p & 0.261462 & 0.000791 & down \\
hsa-miR-6730-3p & 0.15141 & $1.38 \mathrm{E}-06$ & down \\
hsa-miR-2682-3p & 0.141159 & $2.26 \mathrm{E}-13$ & down \\
hsa-miR-7843-3p & 0.073325 & 0 & down \\
hsa-miR-195-3p & 0.061669 & 0.033751 & down \\
hsa-miR-4425 & 0.050224 & 0.044589 & down \\
\hline
\end{tabular}
pathways in cancer and focal adhesion (Fig. 2B). Specifically, 3166 potential targets of miR532-3p were predicted by the miRanda algorithm (Data not shown).

Fig. 2. The significantly enriched GO terms and KEGG pathways of the putative targets of the differentially expressed microRNAs. (A) GO annotation showed that the predicted target genes of the differentially expressed miRs were related to various biological

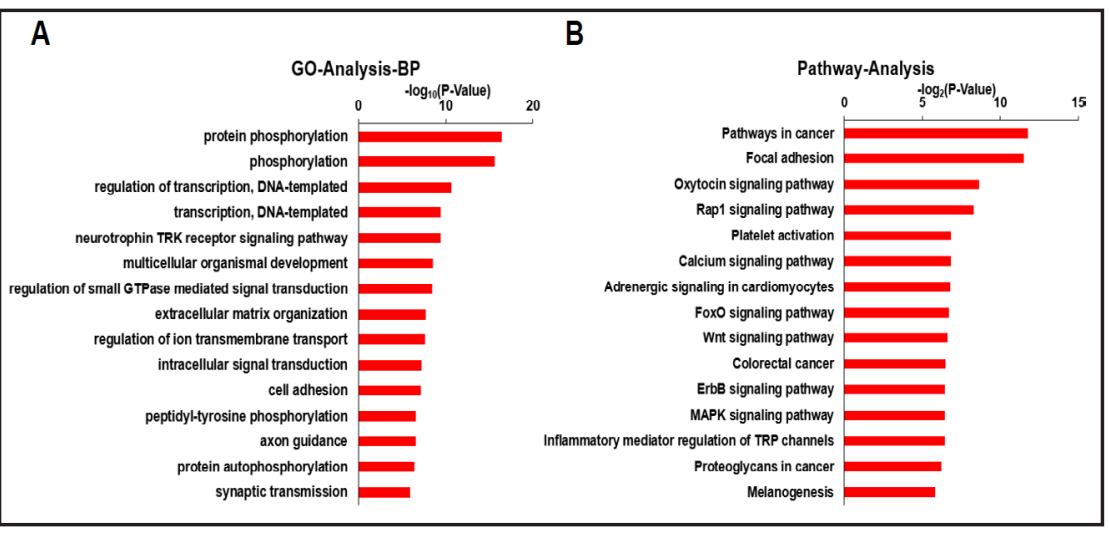
processes (BP). The $\mathrm{x}$ axis showed - $\log$ p-value and y-axis showed the terms of BP. (B) The significant KEGG pathways enrichment in RNA-seq $(\mathrm{p}<0.05)$. The $\mathrm{x}$-axis showed - $\log \mathrm{p}$-value and $\mathrm{y}$-axis showed the KEGG pathways. 
Zhou et al.: 20(S)-Rg3 Inhibits the Warburg Effect in Ovarian Cancer

\section{Validation of the differentially upregulated microRNAs in the 20(S)-Rg3-treated SKOV3} cells

To verify the deep sequencing results, we examined the levels of all the 11 upregulated microRNAs in the 20(S)-Rg3-treated SKOV3 cells and negative control cells by quantitative PCR (qPCR). The results showed the 11 microRNAs were all increased in the 20(S)-Rg3treated SKOV3 cells as exhibited by the deep sequencing data. However, the level of increase of the microRNAs were a little different from the deep sequencing data. miR-3163 was increased the most, followed by miR-324-5p, miR-532-3p, miR-4329, miR-519a, miR-6717-5p, miR-603, miR-664a-5p, miR-486-3p, miR-33a-3p, and miR-1283 was increased the least in 20(S)-Rg3treated cells (Fig. 3).

Upregulated miR-532-3p mediated the inhibitory activity of $20(\mathrm{~S})-\mathrm{Rg} 3$ on the Warburg effect

Since miR-3163, miR-324-5p and miR-532$3 p$ were the top three upregulated miRs shown by qPCR validation assay and the relationship of miR-3163/miR-324-5p to cancer were reported $[8,19,20]$, we chose to study the role of miR$532-3 p$ in the anti-cancer role of $20(\mathrm{~S})-\mathrm{Rg} 3$. We reported that $20(\mathrm{~S})-\mathrm{Rg} 3$ inhibited the Warburg effect in the ovarian cancer cells [10]. Here the influence of $20(\mathrm{~S})-\mathrm{Rg} 3$ on the expression of the

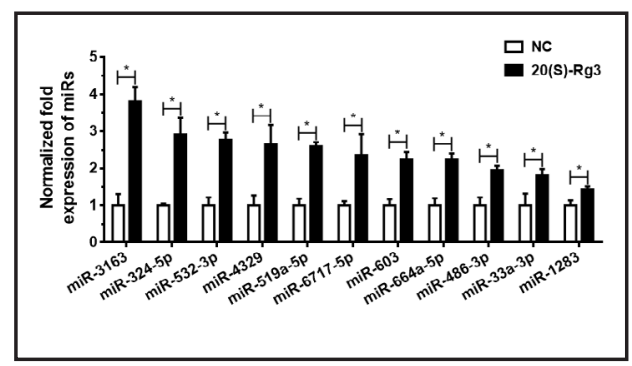

Fig. 3. The validation of the expression levels of the 11 upregulated microRNAs. Relative expression of the 11 upregulated microRNAs was quantified by qRT-PCR. Compared with the control group, miR-3163, miR-324-5p, miR-532-3p, miR-4329, miR-519a-5p, miR6717-5p, miR-603, miR-664a-5p, miR-486-3p, miR-33a-3p, and miR-1283 were significantly increased in the 20(S)-Rg3-treated group. * Indicated $\mathrm{p}<0.05$.

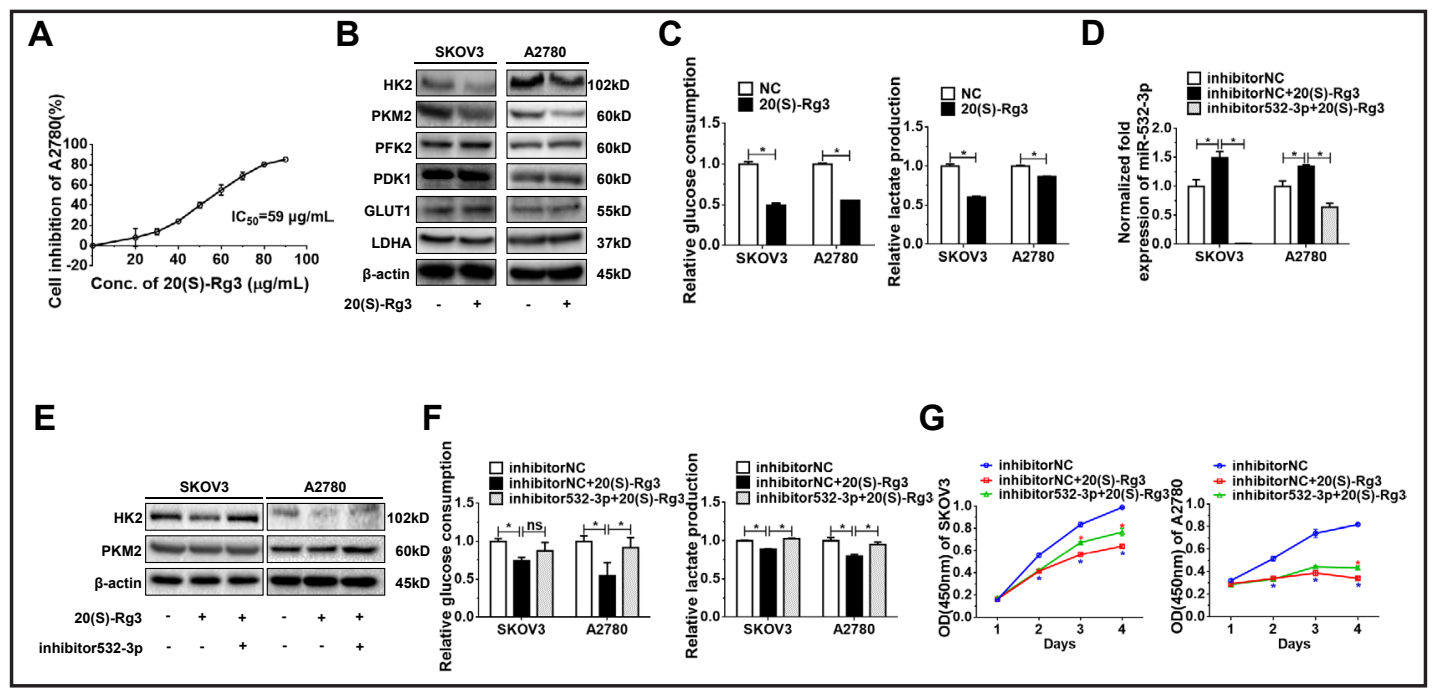

Fig. 4. 20(S)-Rg3 upregulated miR-532-3p to antagonize the Warburg effect. (A) The viability inhibition effect of 20(S)-Rg3 on A2780 ovarian cancer cells after 24h treatment. (B) 20(S)-Rg3 mainly suppressed HK2 and PKM2 expression among other key molecules involved in the Warburg effect. (C) 20(S)-Rg3 weakened glucose consumption and lactate production in both SKOV3 and A2780 cells. (D) 20(S)-Rg3 treatment increased the level of miR-532-3p, which was opposed by transfection of inhibitor532-3p. (E) The inhibition of HK2 and PKM2 expression by 20(S)-Rg3 was reversed by transfection of inhibitor532-3p. (F) 20(S)-Rg3 lessened glucose consumption and lactate production in SKOV3 and A2780 ovarian cancer cells, which was largely recovered by inhibitor532-3p transfection. (G) The retardation of cell growth caused by $20(\mathrm{~S})-\mathrm{Rg} 3$ was partly relieved by miR-532-3p inhibition, especially after 3 days of transfection of inhibitor532-3p. The statistical differences were tested using two-tailed t-test. * Indicated $\mathrm{p}<0.05$. 


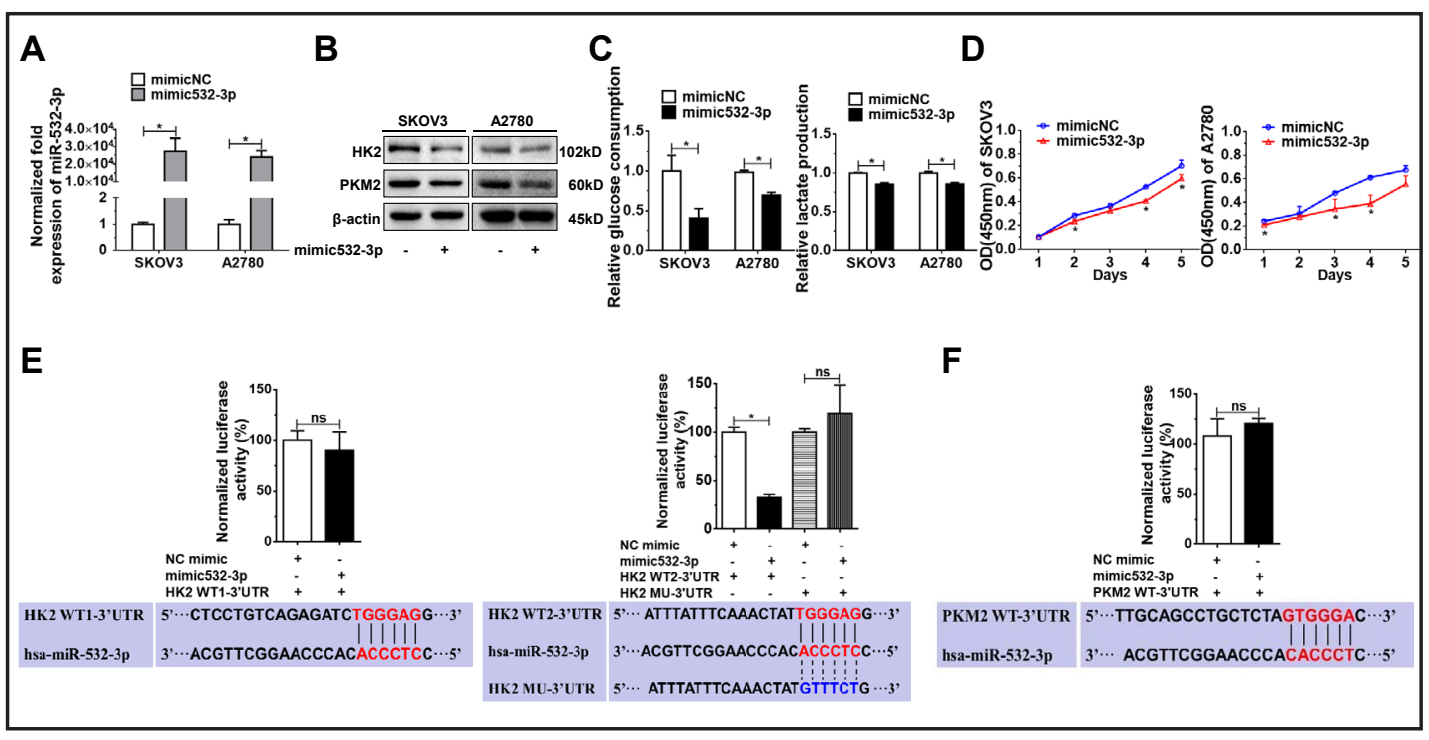

Fig. 5. miR-532-3p targeted HK2 to antagonize the Warburg effect. (A) qRT-PCR showed that miR-532$3 p$ was overexpressed by mimic532-3p transfection. (B) HK2 and PKM2 protein levels were decreased in mimic532-3p-transfected ovarian cancer cells. (C) Glucose consumption and lactate production were reduced in miR-532-3p overexpressed cells. (D) Cell growth of miR-532-3p overexpressed cells was retarded relative to the negative control cells. (E) Luciferase reporter assays showed that miR-532-3p did not bind to the 3'-UTR of HK2 mRNA at the binding site located at the position of 432-436nt, but bound to the binding site located at the 1546-1551nt of the 3'-UTR of HK2 mRNA to inhibit HK2 expression. (F) Luciferase reporter assays showed that miR-532-3p did not bind to the 3'-UTR of PKM2 mRNA at the binding site located at the position of 177-182nt. The statistical differences were tested using two-tailed t-test. The statistical differences were tested using two-tailed t-test. * Indicated $\mathrm{p}<0.05$.

key molecules involved in the Warburg effect was examined. The final concentration of 20(S)$\mathrm{Rg} 3$ was $80 \mu \mathrm{g} / \mathrm{mL}$ for SKOV3 cells as indicated before [21]. IC50 of 20(S)-Rg3 for A2780 cells was determined as $59 \mu \mathrm{g} / \mathrm{mL}$ using CCK8 assay (Fig. 4A), and the final concentration of $40 \mu \mathrm{g} / \mathrm{mL}$ of 20(S)-Rg3 was chosen to be used to treat A2780 cells. Western blotting results showed that 20(S)-Rg3 suppressed expression of HK2 and PKM2 rather than PFK2, PDK1, LDHA and Glut1 (Fig. 4B) in both SKOV3 and A2780 cells. 20(S)-Rg3 weakened glucose consumption and lactate production as well (Fig. 4C). Transfection of miR-532-3p inhibitor into 20(S)-Rg3-treated cells reversed 20(S)-Rg3-induced miR-532-3p upregulation (Fig. 4D), HK2 and PKM2 downregulation (Fig. 4E), glucose consumption and lactate production reduction (Fig. 4F), and inhibition of cell growth (Fig. 4G).

miR-532-3p overexpression by mimic transfection (Fig. 5A) inhibited HK2 and PKM2 expression (Fig. 5B), glucose consumption, lactate production (Fig. 5C) and cell growth of both SKOV3 and A2780 cells (Fig. 5D). HK2 was predicted as a miR-532-3p target by online algorithm, and two putative miR-532-3p binding sites were found in the 3'-UTR of HK2 mRNA sequence. Two wild type luciferase reporter vectors were constructed and each containing one binding site. Luciferase reporter assays showed that the binding site located at the position of 432-436nt in the 3'-UTR was inactive while the binding site located at the 1546-1551nt was responsible for the direct inhibition of miR-532-3p on HK2 expression (Fig. 5E). On the other hand, although no miR-532-3p binding site in the 3'-UTR of PKM2 mRNA sequence was predicted by online algorithm, we manually found one potential binding site of miR-532-3p located in 177-182nt of the 3'-UTR of PKM2. However, luciferase reporter assay did not found the direct suppression of miR-532-3p on PKM2 expression (Fig. 5F). These results indicated that 20(S)-Rg3 upregulated miR-532-3p to directly target HK2 and indirectly inhibit PKM2, and finally antagonized the Warburg effect in ovarian cancer cells. 


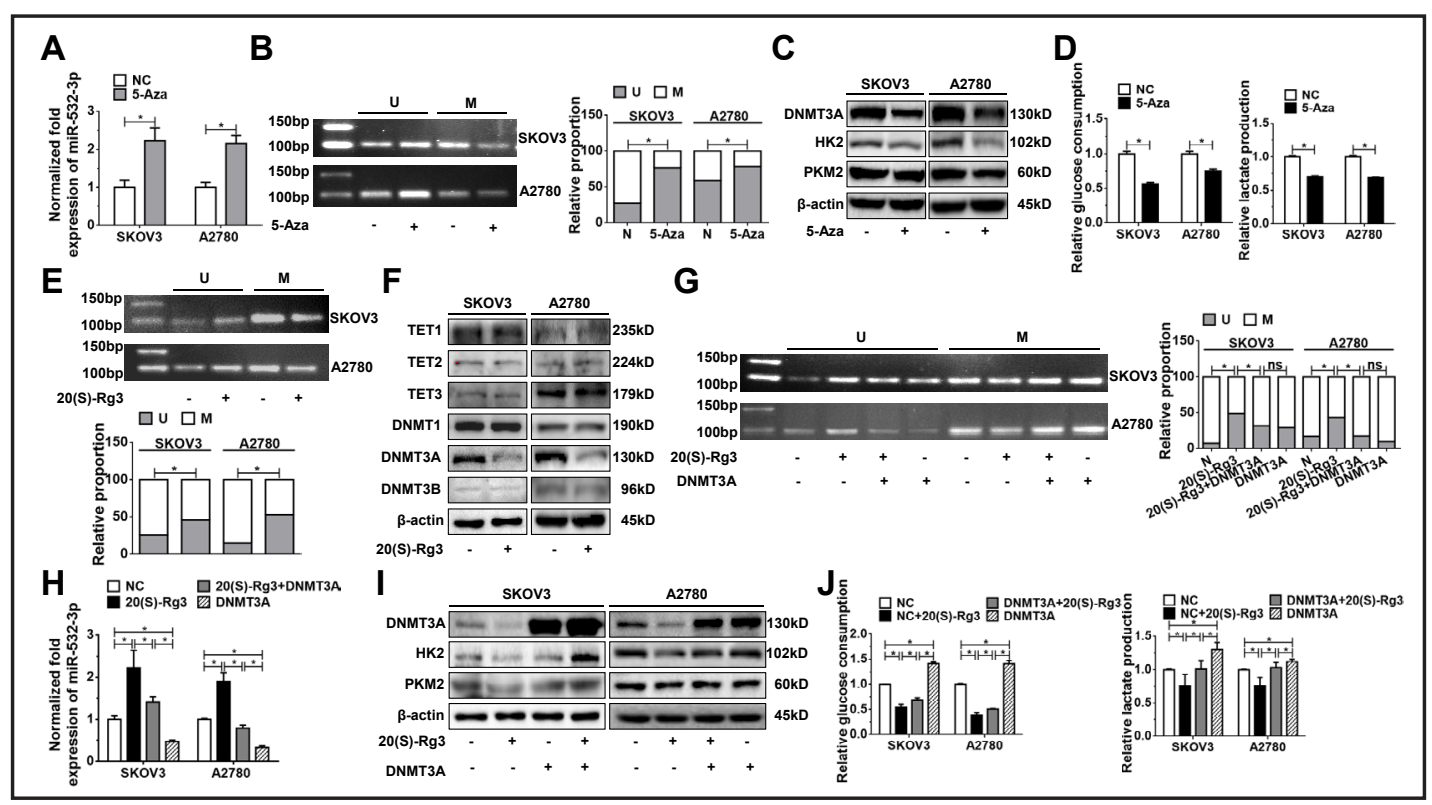

Fig. 6. 20(S)-Rg3 repressed DNMT3A-mediated methylation to upregulate miR-532-3p. (A) miR-532-3p was upregulated by DNA methylation inhibitor 5-Aza at the concentration of $5 \mu \mathrm{M}$ for $72 \mathrm{~h}$. (B) MSP results showed that the proportion of methylated $\mathrm{CpG}$ islands in the promoter region of the host gene of miR532-3p was significantly decreased by 5-Aza. (C) The protein levels of DNMT3A, HK2 and PKM2 were inhibited in 5-Aza-treated SKOV3 and A2780 cells. (D) Glucose consumption and lactate production were reduced by 5 -Aza at the concentration of $5 \mu \mathrm{M}$ for $72 \mathrm{~h}$ in ovarian cancer cells. (E) MSP results showed that the proportion of methylated CpG islands in the promoter region of the host gene of miR-532-3p was significantly decreased by $20(\mathrm{~S})-\mathrm{Rg} 3$ in SKOV3 and A2780 cells. (F) Western blotting results exhibited that DNMT3A was substantially reduced in 20(S)-Rg3-treated cells. 20(S)-Rg3 exerted no obvious influences on the protein levels of DNMT1, DNMT3B and TET1-3. (G) 20(S)-Rg3 increased the unmethylated proportion and decreased the methylated proportion in the promoter region of the miR-532-3p host gene. DNMT3A overexpression reversed the unmethylation effect of 20(S)-Rg3. (H) DNMT3A reduced miR-532-3p in 20(S)-Rg3-treated cells. (I) The inhibition of 20(S)-Rg3 on HK2 and PKM2 expression was antagonized by DNMT3A overexpression. (J) DNMT3A overexpression reversed the inhibition of 20(S)-Rg3 on glucose consumption and lactate production. $\mathrm{U}=$ unmethylated. $\mathrm{M}=$ methylated. The statistical differences of MSP results were tested using two-tailed t-test or Chi-square test. The statistical differences of qRT-PCR and other results were tested using two-tailed t-test. * Indicated $\mathrm{p}<0.05$.

20(S)-Rg3 upregulated miR-532-3p via suppressing DNMT3A-mediated DNA methylation

The mechanism mediating the upregulation of miR-532-3p by 20(S)-Rg3 was further investigated. Since CpG islands in the host gene CLCN5 of miR-532-3p were found using online software (http://www.urogene.org/methprimer/), we studied the involvement of methylation regulation in 20(S)-Rg3-induced miR-532-3p expression. We first used the DNA methylation inhibitor 5-Aza to treat the ovarian cancer cells, and detected over 2-fold increase of miR-532-3p induced by 5-Aza in both SKOV3 and A2780 cells (Fig. 6A). MSP assay results showed the methylation status of the promoter region in the host gene CLCN5 of miR-532-3p was significantly decreased by 5-Aza (Fig. 6B). Additionally, 5-Aza inhibited the Warburg effect in both SKOV3 and A2780 cells illustrated by inhibited HK2 and PKM2 expression (Fig. 6C), glucose consumption and lactate production (Fig. 6D), similar to the effect elicited by $20(\mathrm{~S})-\mathrm{Rg} 3$.

$20(\mathrm{~S})-\mathrm{Rg} 3$ reduced the methylation level of the promoter in the host gene CLCN5 of miR$532-3 p$ as well (Fig. 6E). We then examined the influence of $20(\mathrm{~S})-\mathrm{Rg} 3$ on the expression of the key molecules involved in the DNA methylation process. Western blotting results 


\section{Cellular Physiology Cell Physiol Biochem 2018;45:2548-2559 \\ \begin{tabular}{l|l} 
DOI: 10.1159/000488273 & $\begin{array}{l}\text { O 2018 The Author(s). Published by S. Karger AG, Basel } \\
\text { www.karger.com/cpb }\end{array}$
\end{tabular}}

Zhou et al.: 20(S)-Rg3 Inhibits the Warburg Effect in Ovarian Cancer

displayed substantial decrease of DNA methyltransferase DNMT3A, while no obvious changes in DNA methyltransferase including DNMT1 and DNMT3B and demethylation dioxygenases including TET1/2/3 were observed (Fig. 6F). Hence, the effect of DNMT3A on the level of miR-532-3p was further examined. DNMT3A overexpression reversed the 20(S)-Rg3-restrained methylation of the CpG islands in the promoter region of miR-532$3 p$ host gene in both SKOV3 and A2780 cells (Fig. 6G), resulting in the downregulation of miR-532-3p by DNMT3A overexpression in 20(S)-Rg3-treated cells (Fig. 6H). Consequently, overexpression of DNMT3A antagonized the decrease in HK2 and PKM2 expression (Fig. 6I), glucose consumption and lactate production (Fig. 6J). These results indicated that miR-532$3 p$ expression was negatively influenced by DNMT3A-mediated DNA methylation, which was the key mechanism manipulated by $20(\mathrm{~S})$-Rg3 to promote miR-532-3p-antagonized Warburg effect.

\section{Discussion}

The Warburg effect was one of the metabolic reprogramming hallmarks sustaining the malignant phenotypes of cancer cells. The therapeutic strategy antagonizing the Warburg effect can be exploited for cancer treatment. We have reported that 20(S)-Rg3 exerts antiWarburg effect in ovarian cancer cells by downregulating the key executors of the Warburg effect, HK2 and PKM2. miR has emerged as a class of regulatory molecules involving in various biological processes and functional pathways [22]. Its role in the Warburg effect has been gradually revealed [23-25]. We then investigated the involvement of miR in the anti-Warburg effect of 20(S)-Rg3. 20 differentially expressed miRs were found in 20(S)Rg3-treated cells by deep sequencing technique. Among the upregulated miRs, miR-3163, miR-324-5p, miR-664a-5p, miR-486-3p, miR-33a-3p were reported to be tumor suppressors while miR-603 acted as both oncomir and anti-oncomir [26-30], and the roles of miR-4329, miR-519a-5p, miR-6717-5p and miR-1283 were not defined in cancer [31]. Contrarily, the function of most downregulated miRs including miR-7156-5p, miR-1273e, miR-6730-3p, miR-7843-3p, miR-2682-3p, miR-4425 and miR-195-3p were unclear, except that miR-4634 and miR-4532 were reported to be elevated in cancer tissues or serum [32-34].

Since we aimed to identify the one(s) directly targeting HK2 or PKM2, the 20(S)-Rg3upregulated miRs rather than the downregulated ones were further verified by qPCR. All the 11 upregulated miRs were consistently increased in qPCR assay as in deep sequencing profile. Among the top three upregulated miRs in qPCR results, miR-3163 and miR-324-5p were reported to exert negative roles in cancers [20,35], miR-532-3p was largely undetermined in cancer and was therefore selected to be functionally examined here. As expected, 20(S)Rg3 upregulated miR-532-3p and promoted its direct inhibition on HK2 expression and the Warburg effect. In view that CpG islands were found in the host gene of miR-532-3p and miR-532-3p was upregulated by DNA methylation inhibitor 5-Aza, the effect of 20(S)-Rg3 on molecules controlling DNA methylation was detected. 20(S)-Rg3 somehow specifically repressed DNMT3A without changing the expression of DNMT1, DNMT3B, TET1, TET2 and TET3. 20(S)-Rg3 then relieved DNMT3A-mediated methylation and elevated miR-532-3p. It was the first report that miR-532-3p was under control of DNMT3A-mediated methylation regulation. DNMT3A was reported to be overexpressed in ovarian cancer tissues, inhibiting the anti-oncomir miR-29b and miR-145 expression [36], promising a target for ovarian cancer treatment. The effect of 20(S)-Rg3 on DNMT3A and DNA methylation was consistent with the previous report [37]. Nevertheless, the mechanism about how 20(S)-Rg3 downregulated DNMT3A needed to be clarified.

The mechanisms mediating the inhibition of 20(S)-Rg3 or miR-532-3p on PKM2 have not been revealed here. One of the $20(\mathrm{~S})-\mathrm{Rg} 3$-upregulated miRs possibly targets PKM2, which will be investigated later. Studying the functions of these 20(S)-Rg3-regulated miRs in ovarian cancer would not only provide biofunctional information about the novel miRs but also further the understanding of the anti-cancer mechanism of 20(S)-Rg3. 
Zhou et al.: 20(S)-Rg3 Inhibits the Warburg Effect in Ovarian Cancer

\section{Conclusion}

miRs were manipulated by $20(\mathrm{~S})$-Rg3 to exert the anti-cancer effect in ovarian cancer cells. 20(S)-Rg3 relieved the suppression of DNMT3A-mediated methylation on the expression of miR-532-3p and thus promoted the direct inhibition of miR-532-3p on HK2 to antagonize the Warburg effect. The study provided the new mechanistic evidence about DNMT3A/miR-532-3p/HK2 pathway in the anti-Warburg effect of 20(S)-Rg3.

\section{Acknowledgements}

This work was supported by the National Natural Science Foundation of China (No. 81702576 and No. 30973429), the Natural Science Foundation of Shaanxi Province (No. 2017JM8107) and Foundation of the First Affiliated Hospital of Xi'an Jiaotong University (No. 2016QN-10).

\section{Disclosure Statement}

No Disclosure Statement.

\section{References}

1 Wright AA, Bohlke K, Armstrong DK, Bookman MA, Cliby WA, Coleman RL, Dizon DS, Kash JJ, Meyer LA, Moore KN, Olawaiye AB, Oldham J, Salani R, Sparacio D, Tew WP, Vergote I, Edelson MI: Neoadjuvant chemotherapy for newly diagnosed, advanced ovarian cancer: Society of Gynecologic Oncology and American Society of Clinical Oncology Clinical Practice Guideline. Gynecol Oncol 2016;143:3-15.

- Hanahan D, Weinberg RA: Hallmarks of cancer: the next generation. Cell 2011;144:646-674.

3 Hainaut P, Plymoth A: Targeting the hallmarks of cancer: towards a rational approach to next-generation cancer therapy. Curr Opin Oncol 2013;25:50-51.

4 Cairns RA: Drivers of the Warburg phenotype. Cancer J 2015;21:56-61.

5 Potter M, Newport E, Morten KJ: The Warburg effect: 80 years on. Biochem Soc Trans 2016;44:1499-1505.

6 Liberti MV, Locasale JW: The Warburg Effect: How Does it Benefit Cancer Cells? Trends Biochem Sci 2016;41:211-218.

7 Nag SA, Qin JJ, Wang W, Wang MH, Wang H, Zhang R: Ginsenosides as Anticancer Agents: In vitro and in vivo Activities, Structure-Activity Relationships, and Molecular Mechanisms of Action. Front Pharmacol 2012;3:25.

-8 Kuo WT, Yu SY, Li SC, Lam HC, Chang HT, Chen WS, Yeh CY, Hung SF, Liu TC, Wu T, Yu CC, Tsai KW: MicroRNA-324 in Human Cancer: miR-324-5p and miR-324-3p Have Distinct Biological Functions in Human Cancer. Anticancer Res 2016;36:5189-5196.

-9 Betel D, Wilson M, Gabow A, Marks DS, Sander C: The microRNA.org resource: targets and expression. Nucleic Acids Res 2008;36:D149-153.

10 Li J, Liu T, Zhao L, Chen W, Hou H, Ye Z, Li X: Ginsenoside 20(S)Rg3 inhibits the Warburg effect through STAT3 pathways in ovarian cancer cells. Int J Oncol 2015;46:775-781.

11 Jiang S, Zhang LF, Zhang HW, Hu S, Lu MH, Liang S, Li B, Li Y, Li D, Wang ED, Liu MF: A novel miR-155/miR143 cascade controls glycolysis by regulating hexokinase 2 in breast cancer cells. EMBO J 2012;31:19851998.

-12 Tang H, Lee M, Sharpe O, Salamone L, Noonan EJ, Hoang CD, Levine S, Robinson WH, Shrager JB: Oxidative stress-responsive microRNA-320 regulates glycolysis in diverse biological systems. FASEB J 2012;26:47104721.

13 Bartel DP: MicroRNAs: genomics, biogenesis, mechanism, and function. Cell 2004;116:281-297.

14 Xu W, Zhang Z, Zou K, Cheng Y, Yang M, Chen H, Wang H, Zhao J, Chen P, He L, Chen X, Geng L, Gong S: MiR-1 suppresses tumor cell proliferation in colorectal cancer by inhibition of Smad3-mediated tumor glycolysis. Cell Death Dis 2017;8:e2761.

15 Guo W, Qiu Z, Wang Z, Wang Q, Tan N, Chen T, Chen Z, Huang S, Gu J, Li J, Yao M, Zhao Y, He X: MiR-199a-5p is negatively associated with malignancies and regulates glycolysis and lactate production by targeting hexokinase 2 in liver cancer. Hepatology 2015;62:1132-1144.

16 Taniguchi K, Sugito N, Kumazaki M, Shinohara H, Yamada N, Matsuhashi N, Futamura M, Ito Y, Otsuki Y, 


\section{Cellular Physiology Cell Physiol Biochem 2018;45:2548-2559

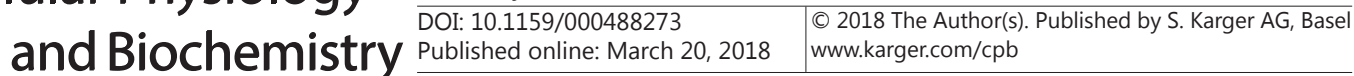

Zhou et al.: 20(S)-Rg3 Inhibits the Warburg Effect in Ovarian Cancer

Yoshida K, Uchiyama K, Akao Y: Positive feedback of DDX6/c-Myc/PTB1 regulated by miR-124 contributes to maintenance of the Warburg effect in colon cancer cells. Biochim Biophys Acta 2015;1852:1971-1980.

17 Nie H, Li J, Yang XM, Cao QZ, Feng MX, Xue F, Wei L, Qin W, Gu J, Xia Q, Zhang ZG: Mineralocorticoid receptor suppresses cancer progression and the Warburg effect by modulating the miR-338-3p-PKLR axis in hepatocellular carcinoma. Hepatology 2015;62:1145-1159.

18 Chen ZX, Mann JR, Hsieh CL, Riggs AD, Chedin F: Physical and functional interactions between the human DNMT3L protein and members of the de novo methyltransferase family. J Cell Biochem 2005;95:902-917.

19 Jia M, Wei Z, Liu P, Zhao X: Silencing of ABCG2 by MicroRNA-3163 Inhibits Multidrug Resistance in Retinoblastoma Cancer Stem Cells. J Korean Med Sci 2016;31:836-842.

20 Su L, Han D, Wu J, Huo X: Skp2 regulates non-small cell lung cancer cell growth by Meg3 and miR-3163. Tumour Biol 2016;37:3925-3931.

-21 Liu T, Zhao L, Zhang Y, Chen W, Liu D, Hou H, Ding L, Li X: Ginsenoside 20(S)-Rg3 targets HIF-1alpha to block hypoxia-induced epithelial-mesenchymal transition in ovarian cancer cells. PLoS One 2014;9:e103887.

22 Drusco A, Croce CM: MicroRNAs and Cancer: A Long Story for Short RNAs. Adv Cancer Res 2017;135:1-24.

-23 Li L, Kang L, Zhao W, Feng Y, Liu W, Wang T, Mai H, Huang J, Chen S, Liang Y, Han J, Xu X, Ye Q: miR-30a-5p suppresses breast tumor growth and metastasis through inhibition of LDHA-mediated Warburg effect. Cancer Lett 2017;400:89-98.

24 Jin F, Wang Y, Zhu Y, Li S, Liu Y, Chen C, Wang X, Zen K, Li L: The miR-125a/HK2 axis regulates cancer cell energy metabolism reprogramming in hepatocellular carcinoma. Sci Rep 2017;7:3089.

-25 Morais M, Dias F, Teixeira AL, Medeiros R: MicroRNAs and altered metabolism of clear cell renal cell carcinoma: Potential role as aerobic glycolysis biomarkers. Biochim Biophys Acta 2017;1861:2175-2185.

-26 Song W, Tang L, Xu Y, Xu J, Zhang W, Xie H, Wang S, Guan X: PARP inhibitor increases chemosensitivity by upregulating miR-664b-5p in BRCA1-mutated triple-negative breast cancer. Sci Rep 2017;7:42319.

27 Bayraktar R, Pichler M, Kanlikilicer P, Ivan C, Bayraktar E, Kahraman N, Aslan B, Oguztuzun S, Ulasli M, Arslan A, Calin G, Lopez-Berestein G, Ozpolat B: MicroRNA 603 acts as a tumor suppressor and inhibits triple-negative breast cancer tumorigenesis by targeting elongation factor 2 kinase. Oncotarget 2017;8:11641-11658.

-28 Guo M, Zhang X, Wang G, Sun J, Jiang Z, Khadarian K, Yu S, Zhao Y, Xie C, Zhang K, Zhu M, Shen H, Lin Z, Jiang C, Shen J, Zheng Y: miR-603 promotes glioma cell growth via Wnt/beta-catenin pathway by inhibiting WIF1 and CTNNBIP1. Cancer Lett 2015;360:76-86.

29 Ye H, Yu X, Xia J, Tang X, Tang L, Chen F: MiR-486-3p targeting ECM1 represses cell proliferation and metastasis in cervical cancer. Biomed Pharmacother 2016;80:109-114.

-30 Han SY, Han HB, Tian XY, Sun H, Xue D, Zhao C, Jiang ST, He XR, Zheng WX, Wang J, Pang LN, Li XH, Li PP: MicroRNA-33a-3p suppresses cell migration and invasion by directly targeting PBX3 in human hepatocellular carcinoma. Oncotarget 2016;7:42461-42473.

31 Bouchareb A, Le Cam A, Montfort J, Gay S, Nguyen T, Bobe J, Thermes V: Genome-wide identification of novel ovarian-predominant miRNAs: new insights from the medaka (Oryzias latipes). Sci Rep 2017;7:40241.

-32 Xu XH, Wu XB, Wu SB, Liu HB, Chen R, Li Y: Identification of miRNAs differentially expressed in clinical stages of human colorectal carcinoma-an investigation in Guangzhou, China. PLoS One 2014;9:e94060.

-33 Shimomura A, Shiino S, Kawauchi J, Takizawa S, Sakamoto H, Matsuzaki J, Ono M, Takeshita F, Niida S, Shimizu C, Fujiwara Y, Kinoshita T, Tamura K, Ochiya T: Novel combination of serum microRNA for detecting breast cancer in the early stage. Cancer Sci 2016;107:326-334.

34 Bibi F, Naseer MI, Alvi SA, Yasir M, Jiman-Fatani AA, Sawan A, Abuzenadah AM, Al-Qahtani MH, Azhar EI: microRNA analysis of gastric cancer patients from Saudi Arabian population. BMC Genomics 2016;17:751.

35 Sun LN, Xing C, Zhi Z, Liu Y, Chen LY, Shen T, Zhou Q, Liu YH, Gan WJ, Wang JR, Xu Y, Li JM: Dicer suppresses cytoskeleton remodeling and tumorigenesis of colorectal epithelium by miR-324-5p mediated suppression of HMGXB3 and WASF-2. Oncotarget 2017;8:55776-55789.

-36 Teng Y, Zuo X, Hou M, Zhang Y, Li C, Luo W, Li X: A Double-Negative Feedback Interaction between MicroRNA-29b and DNMT3A/3B Contributes to Ovarian Cancer Progression. Cell Physiol Biochem 2016;39:2341-2352.

37 Teng S, Wang Y, Li P, Liu J, Wei A, Wang H, Meng X, Pan D, Zhang X: Effects of R type and S type ginsenoside Rg3 on DNA methylation in human hepatocarcinoma cells. Mol Med Rep 2017;15:2029-2038. 